\title{
Ceratectomia fotorrefrativa associada à ceratotomia lamelar pediculada (LASIK) para correção de miopias, com ou sem secagem do estroma
}

\author{
Photorefractive keratectomy associated with lamellarkeratotomy(LASIK) for correction \\ of myopia, with or without drying the stroma
}

Cesar K. Suzuki ${ }^{(1)}$

Edson S. Mori ${ }^{(1)}$

Norma Allemann ${ }^{(1)}$

Paulo Schor ${ }^{(1,2)}$

Mauro Campos ${ }^{(1)}$

Wallace Chamonm ${ }^{(1)}$

\section{RESUMO}

Objetivo: Avaliar os resultados clínicos da ceratectomia fotorrefrativa associada à ceratotomia lamelar pediculada (LASIK), para miopia moderada e alta, com ou sem secagem do leito estromal durante a foto-ablação.

Pacientes e Métodos: Estudo retrospectivo de 39 olhos de 30 pacientes submetidos a LASIK sem secagem do leito estromal durante a fotoablação no período de abril de 1996 a abril de 1997 (Grupo I) e 42 olhos de 28 pacientes com secagem do leito estromal durante a foto-ablação a cada 80 pulsos, no período de abril de 1997 a setembro de 1997 (Grupo II). Após a ceratotomia lamelar pediculada corneana com um microcerátomo automatizado (Chiron Corneal Shaper ${ }^{\circledR}$ ), foi realizada a foto-ablação com excimer laser de fluoreto de argônio de $193 \mathrm{~nm}$ da Summit modelo Apex Plus ${ }^{\circledR}$. Em 2 olhos do Grupo I, a foto-ablação não foi realizada, devido a complicações durante a ceratotomia lamelar.

Resultados: O tempo médio de seguimento pós-operatório foi de 8,7 meses no Grupo I e 7,7 meses no Grupo II. A média de tratamento foi de $-10,81 D( \pm 2,38)$ no grupo I e -8,73D $( \pm 2,82)$ no grupo II. As médias das variações dos equivalentes esféricos obtidos em relação ao tratamento desejado nos meses 1,3, 6 e 12 foram respectivamente, $-0,96 \mathrm{D}( \pm 1,19)$, -1,19D $( \pm 1,37),-1,06 D( \pm 1,41)$ e -1,10D $( \pm 0,66)$ no Grupo I e -0,23D $( \pm 1,02),-0,41 D( \pm 1,34),-0,75 D( \pm 1,16)$ e -1,03D $( \pm 1,31)$ no Grupo II. Três olhos do total $(3,7 \%)$, todos do Grupo I, perderam 2 ou mais linhas de visão. Na visita mais recente, 17 olhos (45,9\%) do Grupo I e 31 olhos (73,8\%) do Grupo II apresentaram Acuidade Visual sem correção de 20/40 ou melhor. Ocorreram 4 complicações intra-operatórias no grupo I, sendo que 2 casos tiveram a foto-ablação não-efetuada e uma complicação intra-operatória no Grupo II.

Conclusão: A secagem do leito estromal possibilitou recuperação visual mais rápida, devido a menor hipocorreção primária. Oseguimento a longo prazo não evidenciou diferenças estatisticamente significantes entre os dois tratamentos.

Palavras-chave: Miopia, cirurgia p.; Laser, cirurgia de; LASIK.

\section{INTRODUÇ̃̃OO}

A ceratotomia lamelar pediculada associada à ceratectomia fotorrefrativa (LASIK), primeiramente descrita por Pallikaris ${ }^{1}$, ganha a atenção do médico oftalmologista a cada dia, em especial devido a algumas vantagens
Serviço de Cirurgia Refrativa

Serviço de Bioengenharia Ocular

Departamento de Oftalmologia, Escola Paulista de Medicina (EPM), Universidade Federal de São Paulo (UNIFESP)

Endereço para Correspondência: A/C Cesar Suzuki R. Botucatu, 824 - São Paulo (SP) CEP 04062-023

Nota Editorial: Pelas análises deste trabalho e por suas anuências sobre a divulgação desta nota, agradecemos a Dra. Marizilda A. Giovedi e ao Dr. José Augusto A. Ottaiano. 
como a recuperação visual precoce e menor número de retornos em relação à ceratectomia foto-refrativa (PRK) para correção de miopias moderadas e altas ${ }^{2-4}$. O estudo minucioso da técnica cirúrgica, das complicações e dos resultados clínicos torna-se imprescindível para que se justifique realizar este procedimento, tendo-se em vista que o PRK é um procedimento com menor custo, além de apresentar maior segurança intra e pós-operatória para baixas miopias.

Algumas etapas da cirurgia de LASIK são seguidas por todos os cirurgiões como: colocação de blefarostato, colocação do anel de vácuo, realização do corte lamelar, foto-ablação e reposicionamento da lamela. Porém alguns detalhes técnicos podem diferir entre os cirurgiões, como a secagem do leito estromal durante a foto-ablação, a irrigação da interface e a programação da foto-ablação. A motivação principal para a utilização da secagem foi a observação do acúmulo de líquido no local da ablação estromal em casos anteriores, podendo ser este um fator a mais para a hipocorreção observada em nossa série de casos.

O presente trabalho tem como objetivo avaliar a influência da secagem do leito estromal durante a foto-ablação na evolução refratométrica dos pacientes, além de descrever complicações intra e pós-operatórias ocorridas.

\section{PACIENTES E MÉTODO}

Todos os pacientes que procuraram espontaneamente o Setor de Cirurgia Refrativa da UNIFESP-EPM foram submetidos a anamnese, medida de Acuidade Visual (AV) com correção atual e sem correção, refratometria estática, biomicroscopia, tonometria de aplanação, mapeamento de retina, videoceratografia computadorizada e paquimetria ultra-sônica. Foram contra-indicados pacientes com doenças sistêmicas de repercussão ocular ou doenças oculares como ceratocone, catarata, glaucoma avançado e retinopatias. A paquimetria foi realizada em todos os pacientes, sendo sempre respeitada uma programação de foto-ablação que preservasse, ao menos, $250 \mathrm{~mm}$ de espessura de leito estromal residual, realizando-se, portanto, hipocorreções no tratamento de alguns casos, após informação e concordância do paciente. Portadores de degenerações periféricas de retina foram avaliados por especialistas e submetidos a fotocoagulação com laser de argônio quando indicado, previamente ao LASIK. Todos os pacientes foram orientados sobre a cirurgia, riscos e possibilidades de complicações, assinando um Termo de Consentimento antes da cirurgia.

O aparelho de excimer laser de fluoreto de argônio de $193 \mathrm{~nm}$ utilizado em todas as cirurgias foi o Summit modelo Apex Plus $^{\circledR}$, e a ceratotomia lamelar pediculada foi realizada com o microcerátomo Automated Corneal Shaper ${ }^{\circledR}$ da Chiron Vision. Para a presente análise foram incluídos pacientes que realizaram a cirurgia para correção do equivalente esférico de pelo menos-5,00D. No período de abril de 1996 a abril de 1997 foram realizadas cirurgias sem secagem do leito estromal
(Grupo I) e no período de abril a setembro de 1997 com secagem do leito estromal (Grupo II). No grupo I, a foto-ablação estromal era realizada após centralização do laser, ocorrendo interrupção somente em caso de perda de fixação do paciente. Após nova centralização o tratamento era continuado até o final. No grupo II, a foto-ablação era interrompida a cada 80 pulsos de laser, sendo realizada neste momento secagem do leito estromal com esponja de Wekcell ${ }^{\circledR}$ na área ablada. Para análise, foram incluídos pacientes com pelo menos um mês de seguimento pós-operatório.

Todas as cirurgias foram realizadas por médicos estagiários do Setor de Cirurgia Refrativa do Departamento de Oftalmologia da Universidade Federal de São Paulo (UNIFESP).

A técnica cirúrgica para realização do LASIK não sofreu modificações até a aplicação do laser, sendo realizada sempre imediatamente após a esterilização do microcerátomo em autoclave, sem luvas, com miose medicamentosa e anestesia tópica. Se a lamela estivesse com boa espessura e tamanho, com leito estromal regular, realizava-se a foto-ablação. Neste momento da cirurgia ocorreu a diferença entre os dois grupos em estudo. No Grupo I foi realizada a foto-ablação de maneira contínua, sem secagem do leito estromal e no Grupo II foi realizada a secagem do leito estromal com esponja de Weckcell a cada 80 pulsos de foto-ablação.

Após o término da foto-ablação, as etapas cirúrgicas foram as mesmas nos 2 grupos, com reposicionamento da lamela, irrigação da interface e acompanhamento da adesão.

Foram padronizadas visitas no $1^{\circ}$ e $15^{\circ}$ dias, além de $1^{\circ}, 3^{\circ}$, $6^{\circ}$ e $12^{\circ}$ meses após a cirurgia. Como a refratometria estática só foi realizada a partir do $1^{\circ}$ mês de pós-operatório, as visitas do $1^{\circ}$ e $15^{\circ}$ dias não foram consideradas para análise. Além da refratometria, foram realizados AV com e sem correção, biomicroscopia, tonometria de aplanação e videoceratografia computadorizada em todos os casos.

A análise estatística da evolução do equivalente esférico pós-operatório dos dois grupos foi realizada com o teste de Mann-Whitney. A distribuição do Delta refracional pósoperatório dos dois grupos foi analisada utilizando-se o teste de Qui-quadrado. Para avaliação de estabilidade refracional pós-operatória ao longo do tempo foi utilizado o teste de Friedmann. As diferenças foram consideradas significativas com $\mathrm{p}<0,05$.

\section{RESULTADOS}

Foram operados 81 olhos de 58 pacientes, sendo $36(62 \%)$ do sexo feminino. Dos pacientes operados, 39 olhos de 30 pacientes foram do Grupo I e 42 olhos de 28 pacientes do Grupo II. A média de idade foi de 30,9 anos, variando de 20 a 47 anos. O tempo médio de seguimento pós-operatório foi de 8,7 meses no Grupo I e 7,7 meses no Grupo II. Excluindo-se 2 olhos do grupo I onde o tratamento foto-ablativo foi abortado devido a uma ceratotomia não adequada, 79 olhos foram incluídos na análise dos resultados clínicos. 
A refração pré-operatória, o tratamento realizado e o Delta Refracional do $1^{\circ}, 3^{\circ}, 6^{\circ}$ e $12^{\circ}$ meses encontram-se na Tabela 1 . A média do equivalente esférico pré-operatório no Grupo I foi de $-11,24 \mathrm{D}( \pm 2,90)$ e de $-8,75 \mathrm{D}( \pm 3,01)$ no Grupo II. A média do tratamento realizado foi de $-10,81 \mathrm{D}( \pm 2,38)$ no grupo I e $-8,73 \mathrm{D}$ $( \pm 2,83)$ no grupo II. O Delta Refracional foi estatisticamente diferente $(p<0,05)$ nos meses 1 e 3 . A Tabela 2 apresenta a distribuição do Delta Refracional em intervalos, na última visita. No Grupo I ocorreram 22 casos (59,5\%) com hipocorreções acima de $1,00 \mathrm{D}$, enquanto que no grupo II ocorreram 12 casos $(28,6 \%)(\mathrm{p}<0,05)$. Todos os pacientes possuíam acuidade visual sem correção $(\mathrm{AVsc})$ pré-operatória pior ou igual a 20/200. Após o tratamento, 17 olhos $(45,9 \%)$ do Grupo I e 31 olhos $(73,8 \%)$ do Grupo II apresentaram AVsc melhor ou igual a 20/40 (Tabela 3$)$.

Houve perda de 2 ou mais linhas em 3 olhos (3,7\%) todos pertencentes ao Grupo I. Nas 81 cirurgias, ocorreram 5 complicações intra-operatórias em cinco pacientes $(6,1 \%)$, das quais duas levaram à suspensão da foto-ablação (tratamento abortado sem seqüelas, ou diminuição da acuidade visual corrigida por óculos), e não foram considerados nos resultados clínicos. Todos os 3 olhos que apresentaram complicações e completaram o tratamento estão incluídos nos resultados clínicos.

Em um caso, o microcerátomo completou a ceratotomia lamelar até o local desejado, porém houve falha no retorno. Neste caso, o microcerátomo foi recuado manualmente, não tendo havido comprometimento do procedimento. Em um olho, ocorreu ceratotomia total (sem pedículo) tendo a lamela da córnea ficado fina e pequena, impossibilitando o tratamento. Em um caso, a ceratotomia foi parcial, impossibilitando a foto-ablação, devido à pouca extensão da lamela. A lipotímia ocorrida em uma paciente, foi uma complicação não relacionada diretamente com o procedimento, porém impossibilitou a foto-ablação completa. Em um caso do Grupo II o microcerátomo parou no início do corte, porém prosseguiu-se o corte, sem comprometer a execução da foto-ablação.

Para a avaliação da estabilidade refracional ao longo do tempo foi utilizado o teste de Friedmann, sendo considerados somente os olhos com todas as visitas pós-operatórias completas $(1,3,6$ e 12 meses). No Grupo I $(n=12)$ as médias do equivalente esférico nos $1^{\circ}, 3^{\circ}, 6^{\circ}$ e $12^{\circ}$ meses foram $-0,73 \mathrm{D},-1,03 \mathrm{D},-1,19 \mathrm{D}$ e $-1,41 \mathrm{D}(\mathrm{p}=0,04)$, enquanto que no Grupo II $(n=12)$ foram respectivamente -0,38D, -0,63D, -089D e $-1,60 \mathrm{D}(\mathrm{p}=0,0014)$.

\section{DISCUSSÃO}

A secagem do leito estromal durante a ceratectomia fotorrefrativa no LASIK tem sido empregada por parte dos cirurgiões refrativos. Este estudo teve como objetivo avaliar a influência desta etapa cirúrgica, adotada no Serviço de Cirurgia Refrativa da UNIFESP-EPM, a partir de abril de 1997, comparado-se com resultados anteriores (abril de 1996 a abril de 1997).

A evolução do equivalente esférico pós-operatório nos Grupos I e II apresentou hipocorreção desde o primeiro mês, maior no Grupo I, em especial nos meses 1 e $3(-0,96 \mathrm{D} \pm 1,19$

\begin{tabular}{|c|c|c|c|c|c|c|c|c|c|c|}
\hline Refração pré** & Tratamento & $\mathbf{n}$ & $\begin{array}{c}\text { Delta } \\
1 \text { Mês* } p<0,05\end{array}$ & $\mathbf{n}$ & $\begin{array}{c}\text { Delta } \\
3 \text { Meses }^{*} p<0,05\end{array}$ & $\mathbf{n}$ & $\begin{array}{l}\text { Delta } \\
6 \text { Meses* }\end{array}$ & n & $\begin{array}{c}\text { Delta } \\
12 \text { meses* }\end{array}$ & $\mathbf{n}$ \\
\hline Grupo I -11,24 $(2,90)$ & $-10,81(2,38)$ & 37 & $-0,96(1,19)$ & 29 & $-1,20(1,37)$ & 30 & $-1,06(1,41)$ & 25 & $-1,10(0,66)$ & 21 \\
\hline Grupo II -8,75 $(3,01)$ & $-8,73(2,83)$ & 42 & $-0,23(1,02)$ & 39 & $-0,41(1,34)$ & 30 & $-0,75(1,16)$ & 29 & $-1,03(1,31)$ & 18 \\
\hline
\end{tabular}

\begin{tabular}{|c|c|c|c|c|c|c|}
\hline & $\begin{array}{c}\text { Menor que } \\
-2,99 D\end{array}$ & $\begin{array}{c}\text { Entre } \\
-2,99 \text { e }-1,01 \mathrm{D}\end{array}$ & $\begin{array}{c}\text { Entre } \\
-1,00 \text { e }+1,00 \mathrm{D}\end{array}$ & $\begin{array}{c}\text { Entre } \\
+1,01 \text { e +2,99 D }\end{array}$ & $\begin{array}{l}\text { Maior que } \\
+2,99 \mathrm{D}\end{array}$ & Total \\
\hline Grupo I & $3(8,1 \%)$ & $19(51,4 \%)$ & $14(37,8 \%)$ & $1(2,7 \%)$ & 0 & $37(100 \%)$ \\
\hline Grupo II & $1(2,4 \%)$ & $11(26,2 \%)$ & $25(59,5 \%)$ & $5(11,9 \%)$ & 0 & $42(100 \%)$ \\
\hline
\end{tabular}

Tabela 3. Acuidade visual no pré-operatório (com correção) e no pós-operatório (com e sem correção) na visita mais recente

\begin{tabular}{|lcccccc} 
& $\begin{array}{c}\text { AVcc pré-op. } \\
\text { Grupo I }\end{array}$ & $\begin{array}{c}\text { AVcc pré-op. } \\
\text { Grupo II }\end{array}$ & $\begin{array}{c}\text { AVsc pós-op. } \\
\text { Grupo I }\end{array}$ & $\begin{array}{c}\text { Avsc pós-op } \\
\text { Grupo II }\end{array}$ & $\begin{array}{c}\text { AVcc pós-op } \\
\text { Grupo I }\end{array}$ & $\begin{array}{c}\text { Avcc pós-op } \\
\text { Grupo II }\end{array}$ \\
$20 / 40$ ou melhor & $28(75,7 \%)$ & $39(92,9 \%)$ & $17(45,9 \%)$ & $31(73,8 \%)$ & $30(81,1 \%)$ & $41(97,6 \%)$ \\
$20 / 60$ a $20 / 100$ & $9(24,3 \%)$ & $2(4,7 \%)$ & $17(45,9 \%)$ & $9(21,4 \%)$ & $5(13,5 \%)$ \\
$20 / 200$ ou pior & 0 & $1(2,4 \%)$ & $3(8,2 \%)$ & $2(4,8 \%)$ & $2(5,4 \%)$ \\
AVsc = acuidade visual sem correção; AVcc= acuidade visual com correção & & $1(2,4 \%)$ \\
\hline
\end{tabular}


comparado com $-0,23 \mathrm{D} \pm 1,02$ e $-1,20 \mathrm{D} \pm 1,37$ comparado com $-0,41 \mathrm{D} \pm 1,34, \mathrm{p}<0,05)$. Isto talvez se deva a uma taxa de ablação menor no estroma mais profundo do que no superficial, com necessidade de adequação do nomograma do LASIK. Com a secagem do estroma, houve uma diminuição da hipocorreção, sendo encontrados 22 casos $(59,5 \%)$ com hipocorreções acima de 1,00D no Grupo I, enquanto que no grupo II ocorreram 12 casos $(28,6 \%)$ na visita mais recente $(p<0,05)$. Consequentemente, foi encontrada uma porcentagem maior de casos entre $\pm 1,00 \mathrm{D}$ no Grupo II (59,5\%) do que no Grupo I $(37,8 \%)(p<0,05)$. CARR et al. ${ }^{5}$ demonstraram, para miopias entre -2 e -7D, hipocorreção de $0,62( \pm 0,78)$ a $0,84 \mathrm{D}( \pm 0,65)$, concordante com o presente estudo. Esta tendência a hipocorreção, particularmente no Grupo I pode explicar a observação de que somente 17 olhos $(45,9 \%)$ do Grupo I apresentaram no pós-operatório AVsc melhor ou igual a 20/40, enquanto que no Grupo II, com a menor porcentagem de hipocorreção, foram encontrados 31 olhos $(73,8 \%)$.

$\mathrm{O}$ estroma da córnea hidratado apresenta lamelas mais distantes, com adsorção de água pelas proteoglicanas constituintes. Tal rearranjo, com modificação intrínseca da matriz extracelular, pode alterar a taxa de ablação corneana, perdendo-se energia com destrução de água. A desidratação por outro lado, deve compactar mais as lamelas, podendo potencializar os tratamentos.

No presente levantamento foram observados 3 casos $(3,7 \%)$ com perda de 2 ou mais linhas com a melhor correção, todos pertencentes ao Grupo I. Zaldivar não observou perda de linha em sua série de pacientes ${ }^{6}$, enquanto que PérezSantonja encontrou $1,4 \%{ }^{7}$ e Salah obteve $3,6 \%$ de perda em 88 olhos $^{3}$.

Nesta série ocorreram 5 complicações intra-operatórias em 81 cirurgias $(6,1 \%)$, todas relacionadas com a ceratotomia lamelar, das quais duas levaram à suspensão da foto-ablação . GIMBEL, em seu levantamento de 53 olhos, observou 5 complicações intra-operatórias $(9,4 \%)$ relacionadas com a ceratotomia lamelar ${ }^{8}$, enquanto Zaldivar encontrou $2,4 \%$ (2 olhos em 83) ${ }^{6}$.

Com relação à estabilidade refratométrica foi observada regressão estatisticamente significante nos dois grupos entre o $1^{\circ}$ e o $12^{\circ}$ mês, utilizando-se o teste de Friedmann $(p<0,05)$. Outros autores também observaram regressão em seus pacientes, sendo que Bas ${ }^{4}$ e Peréz-Santoja ${ }^{7}$ encontraram regressão de $-0,5 \mathrm{D}$ entre o $1^{\circ}$ mês e a visita mais recente.

Apesar de não significante, é interessante notar a maior regressão que ocorreu no grupo com secagem em relaçao ao grupo sem secagem ao longo do tempo. Aparentemente as córneas que inicialmente apresentaram maior tratamento (menor hipocorreção), regrediram mais (Grupo II), o que poderia ser explicado por uma maior formação de colágeno reacional pela maior energia absorvida, ou pela deposição de epitélio em superfícies com maior asfericidade negativa (maior transição), seguindo a tendência natural da córnea retornar a curvatura inicial. Uma das causas da diferença de ablação entre LASIK e PRK deve ser a hidratação estromal. Considerando os resultados obtidos, existe a necessidade de uma correção do nomograma diferenciado para a técnica do LASIK. A confecção deste nomograma deve levar em consideração a hidratação estromal na qual vai ser realizada a ablação, com resultados iniciais hipocorretores para estromas mais hidratados.

\section{SUMMARY}

Purpose: To evaluate the clinical results of LASIK for moderate and high degrees of myopia, with and without drying the stroma during photoablation.

Patient and Methods: Retrospective analysis of 39 eyes of 30 patients submitted to LASIK, without drying the stroma during photoablation, in the period from April 1996 to April 1997 (Group I) and analysis of 42 eyes of 28 patients with drying of the stromal bed at each 80 pulses during photoablation in the period of April of 1997 to September of 1997 (Group II). After lamellar keratotomy with an automated microkeratome (Chiron Corneal Shaper (®)), photoablation with fluoride-argon excimer laser, $193 \mathrm{~nm}$, Summit model Apex Plus ${ }^{\circledR}$ was carried out. In 2 eyes of the Group I, the photoablation was not possible due to complications during the lamellar keratotomy.

Results: The average follow-up was 8.7 months in Group I and 7.7 months in Group II. The mean treatment was $-10.81 D$ ( \pm 2.38$)$ in Group I and $-8.73 D$ ( \pm 2.82$)$ in Group II. The average variations of the achieved spherical equivalent in relation to the attempted treatment in months $1,3,6$ and 12 were respectively, $-0.96 D( \pm 1.19),-1.19 D( \pm 1.37),-1.06 D$ $( \pm 1.41)$ and $-1.10 D( \pm 0.66)$ in Group I and $-0.23 D( \pm 1.02)$, $-0.41 D( \pm 1.34),-0.75 D( \pm 1.16)$ and $-1.03 D( \pm 1.31)$ in Group II. On the last visit 17 eyes (45.9\%) of Group I and 31 eyes (73.8\%) of Group II showed uncorrected VA of 20/40 or better. Four intraoperative complications occurred in Group I, in 2 of them the photoablations were not performed and one intraoperative complication occurred in Group II. There was a loss of 2 or more lines in 3 eyes (3.7\%) ot Group I.

Conclusion: Drying of the stroma led to a faster visual recovery due to smaller hypocorrection. No significant differences were observed in the long-term follow-up.

Keywords: Myopia surgery; Laser surgery; LASIK.

\section{REFERÊNCIAS BIBLIOGRÁFICAS}

1. Pallikaris IG, Papatzanaki ME, Siganos DS, Tsilimbaris MK. A corneal flap technique for laser in situ keratomileusis. Human studies. Arch Ophthalmol 1991;109:1699-702.

2. Brint SF, Ostrick Dm, Fisher C, Slade SG, Maloney RK, Epstein R, et al.. Six-month results of the multicenter phase I study of excimer laser myopic keratomileusis. J Cataract Refract Surg 1994;20:610-15.

3. Salah T, Waring GO, Maghraby A, Moadel K, Grimm S. Excimer laser in 
situ keratomileusis under a corneal flap for myopia of 2 to 20 diopters. Am J Ophthalmol 1996;121:143-55.

4. Bas A, Onnis R. Results of laser in situ keratomileusis in diferent degrees of myopia. Ophthalmology 1998;105:606-11.

5. Carr JD, Stulting RD, Sano Y, Thompson KP, Wiley W, Waring GO. Prospective comparison of single-zone and multizone laser in situ keratomileusis for the correction of low myopia. Ophthalmology 1998; 105:1504-11.
6. Zaldivar R, Davidorf JM, Shultz MC, Oscherow S. Laser in situ keratomileusis for low myopia and astigmatism with a scanning spot excimer laser. $\mathrm{J}$ Refract Surg 1997;13:614-9.

7. Perez-Santonja J, Bellot J, Claramonte P, Ismail MM, Alio JL. Laser in situ keratomileusis to correct high myopia. J Cataract Refract Surg 1997;23:372-85.

8. Gimbel HV, Basti S, Kaye GB, Ferensowicz M. Experience during the learning curve of laser in situ keratomileusis. J Cataract Refract Surg $1996 ; 22: 542-50$.

\section{CONGRESSO BRASILEIRO DE OFTALMOLOGIA}

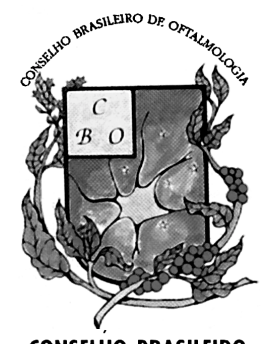

CONSELHO BRASILEIRO DE OFTALMOLOGIA

\section{A 9 DE SETEMBRO DE 2001}

\section{CENTRO DE CONVENÇÕES IMIGRANTES SÃO PAULO • SP}

\section{ATENÇÃO - DATAS IMPORTANTES}

$31 / 01 / 01$ - Data limite para envio de temas livres e pôsteres 30/03/01 - Data limite para envio de Vídeos

Informações atualizadas sobre o evento: www.cbo2001.com.br

Informações: CBO EVENTOS

Al. Santos, 1,343-Cj. 1.110

Telf: (1 1 ) - 3266-4000 / Fax: (1 1 ) $3171-0948$

e-mail: eventos@cbo2001.com.br 